\title{
Dictynna
}

Dictynna

Revue de poétique latine

$1 \mid 2004$

Varia

\section{Blinde Mimesis. Über Ordo und Kontingenz in der literaturgeschichtlichen Traditionsbildung (Horaz und Petron)}

Jürgen Paul Schwindt

\section{(2) OpenEdition \\ Journals}

Édition électronique

URL : http://journals.openedition.org/dictynna/170

DOI : $10.4000 /$ dictynna. 170

ISSN : 1765-3142

Édition imprimée

ISBN : 2-84467-088-1

Référence électronique

Jürgen Paul Schwindt, « Blinde Mimesis. Über Ordo und Kontingenz in der literaturgeschichtlichen Traditionsbildung (Horaz und Petron) », Dictynna [En ligne], 1 | 2004, mis en ligne le 15 novembre 2010, consulté le 11 septembre 2020. URL : http://journals.openedition.org/dictynna/170 ; DOI : https:// doi.org/10.4000/dictynna.170

Ce document a été généré automatiquement le 11 septembre 2020.

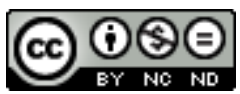

Les contenus des la revue Dictynna sont mis à disposition selon les termes de la Licence Creative Commons Attribution - Pas d'Utilisation Commerciale - Pas de Modification 4.0 International. 


\title{
Blinde Mimesis. Über Ordo und Kontingenz in der literaturgeschichtlichen Traditionsbildung (Horaz und Petron)
}

\author{
Jürgen Paul Schwindt
}

me truncus illapsus cerebro / sustulerat, nisi

Faunus ictum / dextra levasset, Mercurialium /

custos virorum.

(Hor. carm. 2, 17, 27-30)

... et ipse Trimalchio capillatus caduceum tenebat Minervaque ducente Romam intrabat.

(Petron. 29, 3)

1 Es ist zuweilen das Schicksal besonders gut erforschter Texte, daß sie im System der Bezüge, der Quellen und Verweise, das die gelehrte Erklärung über, neben und unter ihnen errichtet hat (Hyper-, Para-, Prae- oder Subtext), zu verschwimmen, konturlos zu werden, ja zu verschwinden drohen. Der Text existiert nur noch im Applikat der mehr oder weniger distinkten Methodik, im Medium seiner philologischen Vermittlung. Die Geschichten, die die gute alte lectura continua der Paraphrase ersetzen sollen, sind zunehmend disparate. Der disiectus membrorum wurde daher seinerseits in den Adelsstand einer strukturgesetzlichen Instanz erhoben ${ }^{1}$. Der Bruch soll Methode haben. Und da man die alte Geschichte doch nicht ganz darangeben möchte, schafft man neue schillernde Vorstellungen von Einheit, zuletzt z. B. den Körper ${ }^{2}$. Jetzt ist der Text ganz diskorporiert, und die Methode ist an seine Stelle getreten.

2 Nun ist gegen diese radikale Form einer substituierenden Exegese solange nichts einzuwenden, wie der Text in der Repräsentation durch seine erklärende Nachzeichnung erkennbar bleibt. Und genau hier liegt das Problem. Die themen- und 
motivgeschichtliche Traktation von Texten hat Rubrizierungen, Indexikalisierungen, Kommentierverfahren zur Folge, die den Text womöglich in eine Paßform zwingen, der er gerade zu entkommen sucht. Das identifikatorische Verfahren, das noch immer den Maßstab für die literaturhistorische Komparatistik liefert, neigt zur exponentiellen Produktion tautegorischer Rede. Anders gesagt : Wenn philologische Erklärung nur Ähnlichkeiten benennt und das tertium der Verwandtschaft schuldig bleibt, versagt sie eben dort, wo Wissenschaft anzuknüpfen hätte. Die mechanische Komparation leistet die Maschine zuverlässiger als menschliche memoria ; nur diese aber kann die Struktur erfassen, die comparandum und comparatum gleichermaßen umgreift. Verwandtschaft gründet womöglich auf Ähnlichkeit jenseits der Themen, Motive und Klänge.

3 Hier lauert gleich die nächste Gefahr, wenn die Grundsätzlichkeit vergleichender Analyse eine Archetypik erzeugt, die sich leicht als umgekehrte Metaphysik der Literaturgeschichte darstellt. Der Weg muß daher ungefähr der folgende sein : Wie in der Textkritik und Textgeschichte der 'Urtext' die Hypostase seiner rectifizierten Erben, mithin eine idealische Konstruktion vorstellt, ohne doch darum seinen historischen Kern preiszugeben, so beschreibt Architextualität ein zugleich text liches und außertextliches Verhältnis. Der Architext ist nicht die Urschrift, von der die anderen Schriften sich herleiten ; er ist der Text, auf den die anderen Texte führen, wenn sie verglichen werden ${ }^{3}$.

4 Ein Fest, ein Unfall, Fortuna, dann Verse. Die Reihe macht Sinn, zuweilen Literatur und sogar Literaturwissenschaft. Sie ist noch kein Archetyp, da sie aus Themen und Motiven gemacht ist, die aber zum Teil schon Strukturen erkennen lassen, die sich archetypisch formieren können : das Fest/der Ritus/die Höhe einer Gestimmtheit, der Blitz/der Un- oder Zwischenfall, der Wechsel/der Umschlag, das Merken/das Schreiben/das Protokoll/der Mythos. Zurück zur thematischen Kette. Und zuerst zu Petron. Das Fest ist die cena; der Sturz wirft einen petauristarius auf den Arm des Trimalchio ; Fortuna, so will man glauben, hat es gut gemeint mit dem Gastherrn ; er schreibt ein Epigramm auf die Göttin $(\S 53,11-55,4)$. Das ist das Gerüst. Noch jeder Kommentator hat auf den Horazischen "Praetext » verwiesen : die cena Nasidieni (sat. 2, 8) mit dem stürzenden Baldachin. Auch hier ist Fortuna im Spiel ; Nomentanus erinnert an ihre unwägbare Macht. Die Parallele ist unabweisbar. Doch was ist gewonnen ? Ein Praetext. Doch was erklärt er ?... neque concipere aut edere partum mens potest nisi ingenti flumine litterarum inundata (Eumolp, \$118, 3) ? Haben Völlerei und Weinseligkeit das poetologische Muster der Satyrika geliefert? Was geschieht mit den Praetexten ? Und überhaupt, sind es Praetexte fürs Schreiben oder fürs Lesen ? Über Letzteres können wir nicht rechten. Unweigerlich stellen sich beim Lesen Praetexte ein. Über Ersteres können wir im Zweifel sein - zumindest über die semiologische Funktion. Werden Praetexte wirklich gelesen und ist ihre Lektüre irgend wichtig für die Verfertigung des neuen Textes ? Unterschätzen wir nicht die Tragweite des Problems. Der lesend schreibende Autor scheint das Idealbild des Philologen. Unser Identifikationsgelüsten wiederholt nur den imaginierten Beutezug des Autors. Schon dies wäre «Blinde Mimesis ", die Versuchseinrichtung williger Unterwerfung unter die erste Gier des benennenden Fest- und Nachmachens.

5 Schauen wir näher hin : Schon Albert Collignon hat die Zonen der Begegnung des Petronschen mit dem Horazischen Text abzustecken gesucht ${ }^{4}$ : Petr. \$26, 9 Trimalchio, lautissimus homo $0^{5}$ soll Hor. sat. 2, 8, 1 Nasidieni... beati ${ }^{6}$ replizieren, Petr. $\$ 40,3$ primae magnitudinis aper an Hor. sat. 2. 8, 6 in primis Lucanus aper erinnern, Petr. §36, 3 ex 
quorum utriculis garum piperatum currebat super pisces Hor. sat. 2, 8, 46 garo de sucis piscis Hiberi anklingen lassen, Petr. 47, 7 castigamus crebris potiunculis risum nach Hor. sat. 2, 8, 63f. Varius mappa compesvere risum / vix poterat gestaltet sein. Nach dem Leitersturz soll Trimalchios - nicht auf die nämliche cena bezogener - Wortwechsel mit Habinnas $\$ 66,1$ 'Quid habuistis in cena ?'... 'Habuimus... in primo porcum' Hor. sat. 2, 8, 4f. Dic, si grave non est, / quae prima iratum ventrem placaverit esca und natürlich wieder V. 6 in primis Lucanus aper nachbilden. Auch der albus anser in Eumolps Dichtung Petr. $§ 93,2$, 3 hätte dann seine Entsprechung im nämlichen Vogelvieh Hor. sat. 2, 8, 88. Schließlich sollen auch der enervierende aetiologische und exegetische Ehrgeiz des gastgebenden Trimalchio sowie die Ausschmückung des Gelages durch kuriose Geschichten in Hor. sat. 2, 8, $92 \mathrm{f}$. (suavis res, si non causas narraret earum et/naturas dominus) bzw. 2, 8, 82f. (dumque / ridetur fictis rerum Balatrone secundo) ihr Vorbild haben. Und vor allem natürlich der Leitersturz $\mathrm{z}^{7}$ und seine pfiffige Verarbeitung in Versen auf Fortuna.

Und was folgt daraus ? "Si ces rapprochements paraissent justes, on est fondé à affirmer que Pétrone avait pour Horace un goût particulier $"^{8}$. Wer wollte widersprechen ? "Entre leurs génies il existe une certaine affinité. Ce ton d'ironie légère, d'élégant persiflage qui règne dans les Satires et les Épitres est celui que prend volontiers Pétrone $»^{9}$. Zweifellos! « Plusieurs des lieux communs dont Pétrone a voulu diversifier son roman ont trouvé déjà dans Horace un humoristique interprète. L' épicurisme mondain, joyeux, d'une morale complaisante et libre, a en quelque sorte pour bréviaire l'œuvre d'Horace $\aleph^{10}$. Wenn ich Collignon recht verstehe, faßt er die 'Anklänge' als Signale für den horizontverschmelzenden Ideentransport. Er interpretiert nicht, nicht den Petrontext und schon gar nicht seine Zwiesprache mit dem Horazischen Text ; er konstatiert Bezüge (Primäridentifikationen), die er in den Horizont eines literarisch-philosophischen Weltbildes einrückt (Sekundäridentifikationen). Neuere Literaturwissenschaft würde Collignon vermutlich unter Einflußforschung subsumieren ${ }^{11}$. Es ist nur folgerichtig, daß die feinfühligsten Interpreten des Petron den Einfluß der Horazischen cena zuletzt deutlich geringer veranschlagt haben. Conte kommt gleich zur Sache : «What matters here is to notice the real difference between Trimalchio's reaction and that of Nasidienus $»^{12}$. Während Nasidienus' Verzweiflung auch durch Nomentanus' Fortunaverse nicht wirklich aufgewogen wird, indem er zuletzt nicht nur Fortuna, sondern auch dem mitleidlosen Spötteln der Gäste ausgesetzt ist, scheint Trimalchio ganz Herr des Geschehens : « he controls not only everything he has programmed but even the unforeseen $»^{13}$.

Diesen Weg hat Victoria Rimell konsequent weiter beschritten : " The inevitability that complete chaos / anti-narrative / episodic patterning is always fake or constructed is never more in evidence than in Sat. 54-5... this little farce is clearly not about freedom, freeing, Fortuna, but about the opposite : control and manipulation of an audience $»^{14}$. Collignons Einflußforschung hätte nun gerade das Gegenteil erreicht : Petron in denkbar weiter Ferne von Horazens Satire. Diese nur Punkt der Abstoßung des Vorbildes und Verkehrung seines 'Sinnes' ins Gegenteil ${ }^{15}$. Nun wird es spannend. Eine Komparatistik, die nicht nach Themen, sondern architextuellen Strukturbildern fragt, könnte im Horaz noch anders fündig werden. Und erschließt uns womöglich eine Dimension, die auch sonst den Satyrika nicht unbekannt ist.

8 « Mit knapper Not ist der Dichter jähem Tod entgangen : auf seinem Gütchen hat ihn ein morscher Baum niederstürzend beinahe erschlagen. Schreck und Empörung sucht nach einem Schuldigen, sich gegen ihn zu entladen... ", so Heinze/Kiessling in der 
Einleitung ihres Komentars zu Gedicht 2, 13 des Horaz ${ }^{16}$. Mit merkwürdiger Renitenz hat sich die Vorstellung von der Lebensechtheit dieses Ereignisses bis heute in der Literatur gehalten ${ }^{17}$. Nicht nur Maurach ${ }^{18}$, auch E.A. Schmidt ${ }^{19}$ zieht den biographischen Kern des Ereignisses nicht in Zweifel. Kiessling und Heinze sind es gewesen, die als erste die Parallele zu Petr. 53, 11-55, 3 gezogen haben : " Das ist die Parodie einer Gattung von epigrammatischer Gelegenheitspoesie, deren lyrisches Seitenstück unser Gedicht darstellt $»^{20}$. Sehen wir zu, ob die Dinge nicht komplizierter liegen.

9 2, 13 setzt ein mit der hyperbolischen Verfluchung des primus inventor, des « ersten Pflanzers » des Baums, dessen Sturz dem Sprecher fast zum Verhängnis geworden sein soll. Die Unverhältnismäßigkeit von Fluch und vorgestelltem Unglück ist sprachlich überdeutlich gemacht : Der Pflanzetag heißt nefastus (1), die Hand des Pflanzers sacrilega (2). Auch sonst läßt es der Dichter an starker Herausstreichung der Merkwürdigkeit des Ganzen nicht fehlen : die Apostrophe (te... arbos, 1/3), tautologisches primum (2), altertümlich-feierliches arbos (3), pathetischer Chiasmus in nepotum / perniciem opprobriumque pagi (3f.) ; die Stimmung setzt sich fort in den übertreibenden Versen 5ff., die den Pflanzer auf eine Stufe mit dem Vatermörder, dem Gastfreundschaftsverletzer, dem Giftmischer stellen. Beachten wir, wie schon hier Befremdlichkeit, ich will nicht sagen, Komik aus dem Umstand erwächst, daß dem Baum in Form einer verkehrten Teleologie nicht geregeltes Wachstum, sondern 'Fallsucht' als organisches Prinzip im Augenblick der Pflanzung unterschoben wird : gepflanzt, nicht um zu wachsen, sondern zu fallen (te, triste lignum, te, caducum, 11). So wenig die Projektion einer Unheilsgeschichte jetzt noch zu nützen vermag, so wenig erreicht die Verfluchung den doch wohl längst verschiedenen Pflanzer. Die Vorstellung, der Pflanzer habe den Baum den Ahnen zum Fluche gepflanzt ${ }^{21}$, ist so abwegig wie die, daß der Fluch den Pflanzer erreichen könne. Die ersten Strophen exotisieren die Vorstellungswelt des Lesers durch die Konstruktion eines grotesken Zeitverhältnisses ${ }^{22}$. Die Strophen 4 und 5 formulieren eine Gesetzlichkeit, die auf den ersten Blick wenig mit den Verwünschungen des Eingangs $\mathrm{zu}$ tun hat. Die Schadensteleologie wird ja nunmehr geradezu in Abrede gestellt. Es gibt keine böse Finalität außer der unbestimmt bleibenden der improvisa (19) hereinbrechenden Gewalt des Todes, die eben dort zupackt, wo man arglos war. Diese Gedankenfigur wenigstens ist im Bilde der drei 'typischen' Mörder vorbereitet. Vater- und Gastfreundsermordung, überhaupt der Giftmord sind Inbegriffe für die nicht erwartete, gleichwohl gut vorbereitete Untat. Nach den Verquertheiten der ersten und mittleren Strophen sind wir auf weitere Finten vorbereitet :

Der Sprecher malt sich aus, was wohl geschehen wäre, wenn ihn der jähe Tod (quam paene, 21) ereilt hätte. Er wäre mit einem Mal in die Unterwelt (furvae regna Proserpinae, 21) gefahren, vor den Richterstuhl des Aiakos getreten (iudicantem vidimus Aeacum, 22) und, wie stillschweigend vorausgesetzt wird ${ }^{23}$, von diesem an die sedes... discretas piorum (23) verwiesen worden, wo er Ohren- und Augenzeuge einer lyrischen Vorstellung durch Sappho und Alkaios geworden wäre, dicht umringt von der bewundernden Menge. Ringsum in der Unterwelt erstarren die Wächterkreaturen und die großen Büßer in staunender Andacht.

11 Zweierlei muß zunächst ins Auge fallen : Die hektische Bewegung der ersten Gedichthälfte, die schäumende Reminiszenz des beinahe gefühlten Schicksalsschlages und die Reflexion auf die reißende Gewalt plötzlichen Verhängnisses schlägt in der Mitte der Ode um in das ruhig entfaltete Bild einer Szene, der die Übel der Welt 
nurmehr zum entrückten Gegenstand dichterischer Kontemplation und kontemplativer Rezeption geworden sind (Aeoliis fidibus querentem / Sappho puellis de popularibus, / et te sonantem plenius aureo, / Alcaee, plectro dura navis, / dura fugae mala, dura belli !, 24-28 $8^{24}$ ). Nicht nur Liebesleid, auch Krieg und Tyrannensturz sind zu sacro digna silentio (29) sediert. Alle Bewegung kommt zur Ruhe : Cerberus läßt - mit leichter Dissonanz zur gesteigerten Aufmerksamkeit (auf das Musische) - die Ohren sinken, und selbst die Schlangen im Haar der Furien, die jetzt bezeichnenderweise Eumeniden heißen, recreantur (36). Prometheus und Tantalus vergessen einmal ihre Fron (dulci laborem decipitur sono, 38), Orion läßt die Löwen- und Luchsjagd ruhen.

Zum zweiten wird man den zeitlichen Status der Szene merkwürdig finden. Der Sprecher wird dessen inne, was ihm um ein Haar beschieden gewesen wäre. Mit verblüffender Sicherheit werden die einzelnen Schritte des imaginierten Hinscheids entwickelt. Proserpina, Aeacus, die Gefilde der Seligen, dann Sappho und Alkaios, die staunende Menge. Alle tun, was sie nach der landläufigen Vorstellung immer tun. Proserpina steht für die Unterwelt, Aeacus richtet, die Lyriker singen, die Hörer lauschen ergriffen. Wenn schon die völlige Übereinstimmung des Bildes mit konventionell gewordenen Begriffen von Unterwelt erstaunlich ist, so wird das Erstaunliche explizit bei der Beschreibung des unseligen Außen der hübschen Genreszene : Das Höllengetier und die großen Büßer tun eben nicht länger, was sie für gewöhnlich und gewissermaßen ex definitione tun ; sie setzen ihre Arbeit aus, staunen, entspannen, lassen sich täuschen und die Geschäfte ruhen. Spätestens mit der an Alkaios gerichteten Apostrophe V. 26f. geht das Beinahe der Katabasis in das Praesens zeitloser Anschauung über. Hierin fügen sich auch die Mirabilien der beiden letzten Strophen. Der absoluten Tempusbeschreibung korrespondiert ihr relativer Status. Vom Standpunkt der imaginierenden Gegenwart wird hypothetisch eine vermiedene Zukunft entfaltet, die in einem doppelten Sinne mit Vergangenheit zu tun hat. Da der Sprecher auf sie als eben noch drohende, doch knapp abgewendete zugleich vorausund zurückblickt, erscheint sie seltsam abgeschlossen, ein Eindruck, der sich verstärkt, wenn man gewärtigt, daß der Blick in die Zukunft in eine Vergangenheit führt, die teils dem Mythos, teils der Literaturgeschichte zugehört.

Wem diese knappe Darstellung der Zeitverhältnisse zu forciert scheint, erinnere sich an den Eingang des Gedichts und das dort entwickelte adynaton eines Fluches, der niemanden mehr treffen, und einer Schadensfinalität, die mitnichten das Werk eines verruchten Pflanzers sein kann. Und er erinnere sich an die zentrale Aporie, daß dem gefährdeten Menschen Unheil gerade nicht von seinem gefahrvollen Tun, sondern von unvorhersehbarer Seite erwächst. Die Vorsorge geht ins Leere, wo Kontingenz sich über die geordneten Bahnen eines menschlichen Lebens hinwegsetzt. Das Böse, als welches das Unverhofft-Plötzliche offensichtlich gedacht ist (: der Vatermord, die Gastrechtsbesudlung, der Giftanschlag), kommt erst in der Konfrontation mit seiner künstlerischen Fixierung (et te sonantem plenius aureo, / Alcaee, plectro dura navis, / dura fugae mala, dura belli !, V. 26-28) zur Ruhe.

Schon der Augenblick des gesteigerten Lebens in der Vergegenwärtigung eines wundersamen Noch-einmal-Davongekommenseins ist seiner poetischen Fixierung posterior. Diese Umkehrung von Anlaß und Folge ist nur die letzte Konsequenz der hier vertretenen Auffassung, wonach die Erfahrung der Kunst erst die Erfahrung des kontingenten Moments ermögliche. Nicht hat die Kontingenz des Baumsturzerlebnisses den glänzenden Ordo einer musischen Existenz heraufgeführt ; nichts kann genau 
genommen von diesem auf jenen führen; vielmehr hat umgekehrt das Bewußtsein der unterweltlichen und überzeitlichen Fortdauer den 'Baumsturz' und seine phantasmagorische Reproduktion ermöglicht. Nur so läßt sich die sichere Deduktion der Unterweltserlebnisse plausibel erklären ; nur so sind die Exzeptionen in Strophe 9 und $10 \mathrm{zu}$ verstehen. Das eigentliche Wunder liegt genau an der Achse des Übersprungs der Anästhesie vom angestammten musischen Bezirk zur umgebenden Hölle. Wenn auch diese erfaßt und in den Strudel unifizierender Bewunderung hineingezogen wird, sind die Voraussetzungen geschaffen für die anästhesierte, künstlerische Freigabe des Baumsturzbildes, das also von hierher, vom Ende, als rhetorischer Anfang, nicht Anlaß des Gedichts verständlich wird ${ }^{25}$.

Das Prekäre der Struktur von 2, 13 ist nicht etwa fehlende Kohärenz der Blöcke 1, 2, 3, sondern die Hinterlastigkeit des Poems, dessen Anfang erst vom Schluß her voll verständlich wird ${ }^{26}$. Die rhetorisch-argumentative Unschärfe, die den Gedichtauftakt charakterisiert, mag man abbildungstheoretisch als blinde Mimesis deuten, als Mimesis, die, ganz auf Verstellung berechnet, ins Leere geht und nur einen Moment zitiert, der seine Dignität ganz aus der Erwartung des Endes bezieht. Blind ist aber auch die Mimesis dieses Endes, das sich auf keine andere fruchtbare Anschauung denn die Kenntnis der Tradition berufen kann.

Was aber hat nun Horaz 2, 13 mit Petron zu tun ? Erinnern wir uns an Contes Feststellung, daß gerade dort, wo der Zufall vorherrschend scheint, nichts dem Zufall überlassen ist. Kontrolle überall. Conte hätte sich schon auf den ingeniösen Beitrag berufen können, den Reinhart Herzog im Herbst 1987 auf der 14. Tagung der Gruppe Poetik \& Hermeneutik vorgestellt hat : «Fest, Terror und Tod in Petrons Satyrica ${ }^{27}$. Das Zauberwort ist die "Kontingenzeliminierung ». Herzog erkennt in der cena Trimalchionis die Strukturen jenes Festtypus, der für viele kaiserliche Veranstaltungen besonders der späteren julisch-claudischen Phase kennzeichnend ist : das Pseudo- oder Zwangsfest. Auch der Erzähler Encolp und seine Verbündeten können dem Zwang zur Satisfaktion der Regieerwartungen nicht entkommen. Sie applaudieren, wenn Applaus, zeigen ihren dégoût, wenn Ablehnung die erwartete Geste ist. Alles auf dem trimalchionischen Fest verläuft nach den Regeln einer diabolischen Organisation. Herzogs Beobachtungen lassen sich in dem von Conte und Rimell verfolgten Sinne ${ }^{28}$ auch auf den Leitersturz ausdehnen. Zeigt sich doch gerade hier, wie unter dem brüchigen Firn des Sturzspektakels die überlegene Regie des Gastgebers waltet. Im Augenblick des Desasters hören wir nicht sofort von Befinden und Reaktion des geschädigten Trimalchio. Wohl aber hören wir die Schreie der Dienerschaft, der Gäste, erfahren von ihren kleinlichen Ängsten und Rücksichten. Dann kommt Trimalchio ins Bild, cum graviter ingemuisset superque bracchium tamquam laesum incubuisset $(\$ 54,2)$. Mit dem einzigen Wort tamquam also gibt der Augenzeuge und Berichterstatter zu erkennen, daß er die Staffage durchschaut, und schildert dann weiter die hektisch akzelerierte Szene, den Auflauf der Ärzte, die in der Manier eines Klageweibs (crinibus passis ; miseram... se atque infelicem proclamavit, §54, 2) herbeistürzende Gattin, den zu Füßen der Symposiasten um Gnade winselnden Gaukler. Wenn Kellers Konjektur per ridiculum das Richtige trifft, macht sich Encolp bereits auf einen weiteren Coup, die Entlarvung des Sturzes als Theaterfall, gefaßt : pessime mihi erat, ne his precibus [sc. petauristarii] per ridiculum $[\mathrm{H}:$ periculo] aliquid catastropha quaereretur $(\$ 54,3)$, sagt er und denkt an die frühere Szene, da ein Koch um ein Haar für ein Versäumnis belangt worden wäre, das sich hernach als wundersame Erfüllung der allgemeinen Erwartungen herausstellte (vgl. §49-50, 1). Argwöhnisch inspiziert der Erzähler den 
Speisessaal, ne per parietem automatum aliquod exiret $(\$ 54,4)$. Die unverzügliche Bestrafung eines Sklaven, der sich bei der Farbe des rettenden Mullverbandes versah, erhöht die Authentizitätssuggestion des Spektakels, das nun freilich genau die von Encolp geahnte paradoxale Wendung nimmt (nec longa aberravit suspicio mea, §54, 5) : Nicht nur entgeht der 'Missetäter' seiner Bestrafung, ein decretum Trimalchios verfügt, daß ihm die Freiheitgeschenkt werde : ne quis posset dicere tantum virum esse servo vulneratum ${ }^{29}(\$ 54,5)$.

Damit ist, nota bene, das Arrangement der Szene noch nicht eingestanden ; es ist Wert darau gelegt, daß die Befreiung noch als Teil der Szene gelesen werde, somit Trimalchio wenigstens vor den minder hellsichtigen Gästen als milde und großzügig erscheinen könne. Es folgt die obligatorische comprobatio durch die Anwesenden und aller lei Geplapper (vario sermone garrimus, $\$ 55,1$ ) über die Unbeständigkeit menschlichen Glückes $^{29}$. Die große Geste ist wiederum dem Arrangeur des Ganzen, Trimalchio, überlassen. Die allgemeinen Reden aufgreifend (ita, §55, 2), bestimmt er - wiederum in der Sprache des symposialen Diktats : non oportet hunc casum ${ }^{30}$ sine inscriptione transire $(\$ 55,2)$, und man beachte, wie sehr auf die Unmittelbarkeit der Abfolge casus - inscriptio Bedacht genommen ist : statim... codicillos poposcit et non diu cogitatione distorta haec recitavit $(\$ 55,2)$. Wo alles arrangiert ist, darf die Reflexion, die auch das heiter-lockere Arrangement nicht entbehren kann, nicht allzu deutlich werden. Das verblüffende Paradox der Szenenpoetik ist, daß mit dem extremen Aufwand der Kontingenzverfertigung (alles soll wie zufällig erscheinen) ein gleicher Aufwand an Kontingenzeliminierung einhergeht (nichts soll dem Zufall überlassen sein).

Das Epigramm codifiziert das eben Geschehene nach dem Gusto des Regisseurs (quod non expectes, ex transverso fit <ubique, /nostra ${ }^{31}>$ et supra nos Fortuna negotia curat. / quare da nobis vina Falerna, puer, \$55, 3). Die interpretatio Trimalchionis soll, fiktionsimmanent, als gültige Deutung des Vorfalls überdauern. Nur vordergründig betrachtet liefert Petron die in nuce gefaßte Genealogie epigrammatischer Dichtung. Mit dem gefälschten Anlaß wird auch die Anlaßdichtung hinfällig. In der Bühnenwelt des Leitersturzes gerät die Epigrammatik zur Karikatur ihrer selbst. Schon deshalb trügt der so naheliegende Vergleich mit der cena Nasidieni. Der feine Spott des überzeichneten Satirenmahls gilt nicht der Doppelbödigkeit des Gesellschaftsspiels, sondern der gravitätischen Ausstellung bohemistischer Nichtigkeiten. Malheur ist nicht Malheur ; und der Gott kommt am liebsten auf der Maschine.

Dagegen ist die strukturell-funktionale Nähe der 'improvisierten' Verschen zu Horaz c. 2, 13 nicht zu verkennen. Nicht nur scheinen sie das Kernstück der Ode : quid quisque vitet, numquam homini satis / cautum est in horas... sed improvisa leti / vis rapuit rapietque gentis (13f. u. 19f.) zu reformulieren ; in Petrons Szene bilden sie den Abschluß des in seinem Schwanken zwischen Wahrheit und Schein hypokritischen Treibens, jenen Abschluß, der in Horazens Ode der Unterweltsszene vorbehalten ist. Die literarische Überhöhung, die bei Petron deutlich Teil des gaukelnden Spiels ist, liefert bei Horaz, wie wir sahen, den Schlüssel zum Verständnis des zwischen Wahrheit und übertreibender Ausschmückung schillernden Eingangs.

In der cena Nasidieni werden die Reaktionen auf den Sturz des Baldachins nicht ausdrücklich und reliefartig als Dichtung abgesetzt. Schon deshalb ist Zurückhaltung bei autoreferentiellen Deutungen geboten. Petron hat die regelrechte mise en abyme, als deren Entsprechung in der komplizierteren Form der Inversion die Ode 2, 13 gelten kann. Hier wird die Beziehung von Ereignis und Dichtung nicht narrativiert, sondern 
das Dichtungserlebnis als hypothetisch sichere Folge eines hypothetischen Ereignisses vorgestellt ${ }^{32}$. Die Sicherheit, mit der das Hypotheton des Dichtungserlebnisses entwickelt wird, läßt, wie beschrieben, keinen anderen Schluß zu, als daß die Urerfahrung der Dichtung der Erfahrung eines besonderen Augenblicks vorausgegangen ist. Ohne das apriorisch gesetzte Fundament einer poetischen Lebensund Unsterblichkeitsgewißheit wird man nicht ernsthaft nach einer Wahrheit der Beinahe-Erfahrung fragen. Die mise en abyme des Horazgedichtes ist nicht, wie im Petron, das Schlußstück einer Geschehensfolge, die so noch einmal in der poetischen, reflexiven Zuspitzung als Ganze erscheinen kann, sondern Begründung eines Anfangs, der dem Erzählten vorausliegt ${ }^{33}$. Die strukturelle Gemeinsamkeit der Texte liegt nun freilich darin, daß die mise en abyme in hochartifizieller Weise getarnt ist : Bei Petron ist sie Teil der Betrugsgeschichte, konsequent im Modus der Ableugnung von Berechenbarkeit und Ordnung verfaßt ; Horazens Täuschung wird erst im Ende der zugleich vergangenheits-und zukunftsgewissen Prospektion erkennbar. 2, 13 ist ganz auf Kontingenzreduktion gestellt. Der paradoxe Effekt ist nur, daß sie sich nicht wirklich bannen läßt oder nur um den Preis, daß durch Umkehr des Anlaß-FolgeVerhältnisses jegliches Vorkommnis in einen symbolischen Bezugszusammenhang integriert werden muß. Schon die Konstruktion der bösen Teleologie des Baumes ist der Versuch der Beschwichtigung und Abmilderung des jäh hereinbrechenden Verhängnisses ${ }^{34}$. Gleiches gilt von den gnomischen Sätzen, die aus der Not imperativischer Kontingenz eine Regel zu ziehen suchen. In einer für viele Horazische Oden charakteristischen Bewegung weist die Unterweltsszene, in der Glück und Grauen in geregelten Bahnen verlaufen, am Schluß über den implizierten ordo hinaus. Sapphos und Alkaios' Lieder ziehen nicht nur die Frommen an ihren sedes discretae in ihren Bann ; auch Cerberus, die Nattern im Haar der Eumeniden, Prometheus, Pelops und Orion lauschen ergriffen, und indem sie dies tun, hören sie auf zu sein, was sie sind : wenn sie das Geschäft ruhen lassen, mit dem sie ex definitione verbunden sind. Die Verkehrung ins Gegenteil ist ein Zug, der sich an jedem der drei Gedichtblöcke beobachten läßt. Es wäre nur konsequent, wenn solche Vertauschung für die Gedichtstruktur im ganzen konstitutiv wäre ${ }^{35}$.

21 Wie sehr Anfang und Ende der Ode aufeinander bezogen sind, wird auch aus folgendem deutlich : Am Anfang die Figur der Vermeidung plötzlicher Auslöschung, am Ende die Auffüllung des Gerade-nicht-Erfahrenen mit Bildern der Intensität, auch dies ein substantieller Verweisungszusammenhang. Daß es das knapp Vermiedene ist, das um ein Haar das erfüllte Bild einer befriedeten Unterwelt heraufgebracht hätte, gehört zu den noch unerwähnt gebliebenen trügerischen Konditionen des Gedichts ${ }^{36}$. Der Trug des Anfangs scheint noch einmal im Ende auf, wenn die Erzbetrüger Pelops und Prometheus sich willig über ihre gerechten Qualen hinwegtrügen lassen. Der Trug ist eine Funktion von Dichtung. Und nicht nur das. Als absichtslose Täuschung steckt er auch im Kern der Mimesis, die das, auf dessen Abbildung sie ausgeht, notwendig verfehlt. Zumal dann, wenn es um die Darstellung blinder Gesetzmäßigkeit geht. Die schädlichen Antriebe des Unheilsbaumes, der Sturz, die Gründe für die Errettung werden verborgen in einer mythhistorischen Archäologie (in c. 2, 17, 27-30, ist es Faunus, dem der Mercurialis vir seine Rettung verdanken will), das blinde Gesetz blinder Zufallsmächte ist unergründlich, die Zukunft des geretteten Sprechers liegt verborgen in einer großen Vergangenheit. Der Sprecher hat nichts gesehen. Quam paene vidi... Seine Mimesis geht ins Leere. Als blinde gibt sie zu sehen nur vor. 
Blind ist auch die Mimesis des täuschenden Trimalchio (sein Konterfei im Wandgemälde am Eingang seines Hauses führt den Merkursstab, caduceus, $\$^{37}, 3^{38}$ ). Sie verfehlt das Ereignis doppelt - nach der Sache (sie beschreibt nichts) und nach dem Sinn (sie verfälscht ihn). Aber die Fälschung macht auch, daß die blind zu sein vorgebende Mimesis am Ende doch das Richtige trifft. Auch im Verhältnis zur Tradition, die sie gerade dort nicht verleugnen kann, wo sie nicht in konventionellen Bildern und Szenen reminisziert wird. Der Architext der Leitersturzszene ist eben jene trügerische Erzählung, die schon Horaz c. 2, 13 fundierte. Es ist die Geschichte von der Parthenogenese der Dichtung, die keiner anderen Erzählung als ihrem eigenen gleichmäßigen Sprechen verpflichtet ist. Man trifft sie an verschiedenen Orten zu verschiedenen Zeiten. Eine Tradition ist das noch nicht. Es müßte denn sein, sie erschiene für einen Augenblick in der neuen Totale eines neuen Gedichts.

\section{NOTES}

1. Man denke nur an F. I. Zeitlins Studie : Petronius as Paradox : Anarchy and Artistic Integrity, TAPhA 192, 101, S. 631-684.

2. Siehe V. Rimell, Petronius and the Anatomy of Fiction, Cambridge 2002.

3. Der Genettesche Terminus (Introduction à l'architexte, Paris 1979) wird, wie der Leser längst bemerkt haben wird, seiner 'ursprünglichen' Bedeutung entfremdet. Es ist kein primär generologisches, auch kein nur diskursanalytisches Problem, das zur Verhandlung steht.

4. A.C., Étude sur Pétrone. La critique littéraire, l'imitation et la parodie dans le Satiricon, Paris 1892, S. $255 f$.

5. Die Zitate nach K. Müllers Teubneriana, Stuttgart/Leipzig ${ }^{4} 1995$.

6. Die Zitate nach D.R. Shackleton Baileys Teubneriana, Stuttgart 1985.

7. In einer geistreichen Wendung von J.P. Sullivan, The Satyricon of Petronius. A Literary Study, London 1968, direkt auf die cena Nasidieni zurückgeführt : "Oddly enough, the change from awning to acrobat may have been suggested to Petronius by a conscious or unconscious verbal memory. Balatro, comforting his host, enumerates some of the mishaps that may befall a dinner party : adde hos praeterea casus, aulaea ruant si / ut modo ; si patinam pede lapsus frangat agaso (71-2), with which cf. puer... delapsus est (54.1)» (S. 126 Anm. 4).

8. Ebd., S. 258.

9. Ebd.

10. Ebd.

11. Hierher gehören auch die formgeschichtliche Untersuchung von J. Révay, Horaz und Petron, CPh 17, 1922, S. 202-212, L.R. Shero, The « Cena » in Roman Satire, CPh 18, 1923, S. 126-143, und im Prinzip auch noch M. T. Rodriquez, La presenza di Orazio nella cena Trimalchionis, AAPel 57, 1981, S. 267-280, M. Coccia, Cena di Nasidieno e cena di Trimalchione, in : R. Uglione (Hrsg.), Atti del convegno nazionale di studi su Orazio, Turin 1992, S. 131-148, u.E. Courtney, A Companion to Petronius, Oxford 2001, S. 99.

12. G.B. Conte, The Hidden Author. An Interpretation of Petronius' Satyricon, Berkeley/Los Angeles/London 1996, S. 130.

13. Ebd., S. 131.

14. Ebd. (Anm. 2), S. 191f. 
15. Die cena Nasidieni gibt der Horazerklärung - ganz unabhängig von möglicher Fortwirkung große Probleme auf. Eine ansprechende mikro- und makrostrukturelle Ausdeutung des Baldachinsturzes unternimmt R. R. Castin, The Fall of the Curtain (Horace S. 2.8), TAPhA 127, 1997, S. 233-256. S. auch E. Oliensis, Ut arte emendaturus fortunam : Horace, Nasidienus, and the Art of Satire, in : T. Habinek / A. Schiesaro (Hrsg.), The Roman Cultural Revolution, Cambridge 1997, S. 90-104, bes. S. 99-102.

16. Zitiert nach der 8. Aufl., Berlin 1955, S. 209.

17. Mitunter sogar in der forcierten Lesart, daß die Baumsturzepisode als 'Höhepunkt' eines - in der Nachbürgerkriegszeit - ereignisarmen Lebens herhalten muß. So bei H. Hommel, Horaz. Der Mensch und das Werk, Heidelberg 1950 : «Von da an verlief Horaz' Leben... mehr und mehr in ruhigen Bahnen, so daß etwa der Unfall, bei dem er auf seinem Landgut beinahe durch einen umstürzenden Baum erschlagen wurde..., zum bedeutenden, immer wieder erwähnten Ereignis ward » (S. 116).

18. G. Maurach, Horaz. Werk und Leben, Heidelberg 2001. Dort, S. 158-160, unter der Überschrift 'Biographische Notiz : C. 2, 13 und 2, 17' : "Über Biographica aus den Jahren zwischen dem Geschenk des Landgutes und der Publikation der ersten Oden-Sammlung... wissen wir so gut wie nichts.... Nur dies erkennt man, dass über ihm... große Gefahr gehangen hatte :... Horaz wäre beinahe von einem umstürzenden Baum erschlagen worden.... Wann dieser Sturz des Baumes... geschah, ist schwer zu sagen » (S. 158f.).

19. E. A. Schmidt, The Date of Horace, Odes 2.13, in : Vir bonus discendi. FS O. Skutsch, hrsg. v. N. Horsfall, London 1988 (= BICS, Suppl. 51), S. 118-125 (vgl. auch : E.A.S., Der Wechsel zur Musendichtung, in : ders., Zeit und Form. Dichtungen des Horaz, Heidelberg 2002, S. 175-189).

20. Ebd. (Anm. 16), S. 210. Wenige sind ihnen gefolgt. Etwa F. Arnaldi in seiner kommentierten Ausgabe der Horazischen Oden und Epoden, Messina 1940, S. 135. Vgl. auch R.G.M. Nisbet u.M. Hubbard, A Commentary on Horace : Odes. Book II, Oxford 1978, S. 202.

21. Neues Material zum topischen Hintergrund jetzt bei K. Yanagisawa, The 'Parentage' Topos. Horace 2, 13. 1-12 and Ovid, Amores 1, 12, MH 57, 2000, S. 270-74.

22. Wie sehr dem Sprecher daran gelegen ist, « nicht so sehr das schreckliche Ereignis selbst, wie vielmehr seine Beurteilung (crediderim) desselben zum Thema [zu machen] », hat schon E. Dönt beobachtet (Horaz II 13, in : Arktouros. FS B. M. W. Knox, hrsg. v. G. W. Bowersock, W. Burkert u. M.C.J. Putnam, Berlin/New York 1979, S. 413-18, dort S. 414).

23. Doch ist der Bezug auf V. 12 domini... immerentis plausibel (E.A. Schmidt, Sabinum. Horaz und sein Landgut im Licenzatal, Heidelberg 1997, S. 103).

24. E. Schäfer, Horaz nach Actium, WJA 13, 1987, S. 195-207, hat feinsinnig den strukturellen Zusammenhang des « Gedicht[s] im Gedicht » mit dem rahmenden Anlaß beobachtet. Nur glaube ich, daß man nicht schließen darf : « Der Inhalt der äolischen Lieder... ist das Leben der Sänger selbst, und nicht anders ist es bei Horaz, der hier etwas wirklich Erlebtes zu Gehör bringt » (S. 198). Vielmehr markiert - umgekehrt - die mise en abyme der lesbischen Lieder die wesentliche Literarizität des 'Rahmens'.

25. Ich möchte diese radikale Deutung der Gedichtstruktur für kompatibel halten mit der gemäßigteren Auffassung, die G. Davis in seinem Buch Polyhymnia. The Rhetoric of Horatian Lyric Discourse, Berkeley/Los Angeles/Oxford 1991, zu der Formel vom 'autobiographical mythos' verdichtet hat. Über die Authentizität des Ereignisses kann nicht ernstlich entschieden werden.

26. Das brutum factum der Hinterlastigkeit des Gedichts ist seit F. Klingners Analyse weithin anerkannt (Ohnmacht und Macht des musischen Menschen, in : ERMHNEIA. Festschrift 0. Regenbogen, Heidelberg 1952, S. 119-26, bes. S. 125f.).

27. Erschienen in : Das Fest, hrsg. v. W. Haug u. R. Warning, München 1989 (= Poetik und Hermeneutik XIV), S. 120-150.

28. S. oben Anm. 12 u. 14 (bzw. 2). 
29. Schon hier ist das psychosozile Phänomen der Streßkompensation durch Versprachlichungsprozesse in treffender Knappheit narrativiert.

30. C. Connors, Petronius the poet. Verse and literary tradition in the Satyricon, Cambridge 1998 , hat auf den Doppelsinn des Wortes hingewiesen : « A conventional metaphor in the word casus regularly associates happenstance with the metaphor of falling; this conventional metaphor is made literal and concrete in the acrobat's fall » (S. 53). Wenn sie weiter folgert, daß « in effect, the actual 'threat' posed by the fall of the acrobat is tamed by being transformed into metaphor, and at the same time, the conventional metaphors of falling are enlivened by the acrobat's mishap " (ebd.), so sollte sich auch diese Beobachtung mit dem Phänomen der Kontingenzelimierung zureichend beschreiben lassen - einer Kontingenz freilich, die paradoxerweise dadurch 'gezähmt' wird, daß sie dem Oberbegriff der Fortuna unterstellt wird.

31. Add. Heinsius ; anders E. Campanile, Letture Petronianae, SIFC 46, 1974, S. 41-50. Er läßt auf et $<$ sua $>$ folgen (S. 46).

32. Vgl. eine Beobachtung E. Schäfers über den modalen Status der Gedichthälften : "Die scheinbare Wirklichkeit der ersten Hälfte wird durch paene eindeutig zur Möglichkeit, während der durch paene als nur möglich eingeführte Besuch der Unterwelt in der zweiten Hälfte den Anschein des Wirklichen gewinnt » (ebd. [Anm. 24], S. 197f.).

33. In diesem Sinne darf ich mich der substantiellen Deutung E. A. Schmidts anschließen : « 'Ille et nefasto te posuit die' stellt die Entstehung der horazischen Lyrik dar, gibt ihre Aitiologie » (ebd. [Anm. 23], S. 104).

34. Darin ähnlich dem sprachlichen Effekt, den S. Commager so beschrieben hat : « By blowing up a trivial event to absurd dimensions he seems to seek relief from it by assuring himself of its triviality " (The Odes of Horace. A Critical Study, New Haven/London 1962, ${ }^{2} 1963$, S. 140). « Bereits die Überzeichnung », so H. P. Syndikus, « ist also ein Mittel heimlicher Distanzierung » (Die Lyrik des Horaz. Eine Interpretation der Oden, Bd. 1, Darmstadt ${ }^{3} 2001$, S. 415).

35. Hier sei wiederum an die wirkungsmächtige Darstellung F. Klingners erinnert, der im Horizont seiner thematologischen Grundannahmen verpflichteten, idealistisch glättenden Interpretation mit einfachen Strichen die gedichtimmanente Verwandlungsbewegung nachgezeichnet hat : « ... der Anfang ist erst als äußerster Gegensatz vom Ziel aus ganz zu begreifen. Denn was geschieht denn in dem Gedicht, wenn man es als Vorgang, als Bewegung auffaßt, wie es doch offenbar verstanden sein will ? Etwas verwandelt sich in sein Gegenteil - um es mit den allgemeinsten Worten zu sagen » (ebd. [Anm. 26], S. 125).

36. Hierher zähle ich nicht die hübsche, dabei wenig überzeugende Formel, auf die R. Minadeo die heikle Balance der Gedichthälften gebracht hat : "Imagistically, the lignum caducum dominates the first half, while the golden plectrum is the very cynosure of the second. Both phallic emblems, their correspondance invests the ode with its final shape and meaning " (The Golden Plectrum. Sexual Symbolism in Horace's Odes, Amsterdam 1982, S. 223).

37. Für Heinsius' Alternativkonjektur zum überlieferten liberatum : verberatum tritt mit Gründen G. C. Giardina ein (Note a Petronio, MCr 5-7, 1970/1972, S. 178-187, dort S. 180f.).

38. Zur Ausdeutung des piktural begleiteten introitus s. J. Bodel, Trimalchio's Underworld, in : J. Tatum (Hrsg.), The Search for the Ancient Novel, Baltimore/London 1994, S. 237-259, bes. S. 243-248. - Hier liegen selbstredend unausgeschöpfte Möglichkeiten zur Konstruktion einer Architextualität korrespondierender Bild- und Symbolwelten. Die Merkurischen Männer wollen in große Gefahr geraten und aus dieser glücklich errettet worden sein. Und weiters : Daß Encolpius den links vom Eingang des Trimalchionischen Hauses postierten Bild-Hund zunächst für echt hält, möchte man der gedichtanfänglichen Täuschung über die Authentizität des Baumsturzes vergleichen. Wenn der Hund am Ende doch leibhaftig den Gästen zu Leibe rückt, hat sich eine Metamorphose ereignet (in diesem Sinne Herzog, ebd., S. 125 mit Anm. 33), die sich womöglich als Karikatur unseres Zweifels über den Status der Fallholz-Geschichte lesen läßt. 


\section{AUTEUR}

JÜRGEN PAUL SCHWINDT

Universität Heidelberg 